\title{
Validation of subordinal names for "Systema Angiospermarum"
}

\author{
ALEXEY SHIPUNOV ${ }^{1} \&$ JAMES L. REVEAL ${ }^{2}$ \\ ${ }^{1}$ Department of Biology, Minot State University, 500 W University Ave, Minot, ND 58707, U.S.A.; email: dactylorhiza@gmail.com \\ ${ }^{2}$ L.H. Bailey Hortorium, Department of Plant Biology, 412 Mann Library Building, Cornell University, Ithaca, New York 14853-4301, \\ U.S.A.; email: James L. Reveal, jlr326@cornell.edu
}

\begin{abstract}
Nineteen subordinal names for flowering plants are established for the online version "Systema Angiospermarum."
\end{abstract}

Key words: Angiospermae, suprageneric names, suborders, validation

Alexey Shipunov's "Systema Angiospermarum," started in 1991, is a constantly revised on-line alternative to the APG classification (APG III 2009) that is based, since 2003, on a taxonomic synthesis published by Shipunov (see also http://herba.msu.ru/shipunov/ang/ang-en.htm). The current version is number 5.1 (http://herba.msu.ru/shipunov/ang/current/syang.pdf—dated 10 November 2010), and like previous editions, it will continue to undergo modification. One of the features of "Systema Angiospermarum" is its detailed hierarchical approach. Following Takhtajan $(1997,2009)$ in particular, the classification emphasizes superand suborder ranks as the fundamental units of classification. However, since 2003, several new suborder names were adopted that, until now, were not validly published. Accordingly, the following names are established.

Alseuosmiineae Shipunov, nom. nov., based on Alseuosmiaceae Airy Shaw in Kew Bull. 18: 249. 8 December 1965.-Type: Alseuosmia A. Cunn. (1838).

Amborellineae Shipunov, nom. nov., based on Amborellales Melikyan, Bobrov \& Zaytzeva in F.Manitz \& F.H.Hellwig, 14 Symp. Biodiv. Evolutionsbiol.: 122. 1 September 1999.-Type: Amborella Baill. (1873).

Aquifoliineae Shipunov, nom. nov., based on Aquifolieae DC., Prodr. 2: 3. Nov (med.) 1825.-Type: Aquifolium Mill., nom. illeg. (1754).

Brassicineae Shipunov, nom. nov., based on Brassiceae DC. in Mém. Mus. Hist. Nat. 7(1): 242. 20 April 1821.-Type: Brassica L. (1753).

Dipsacineae Shipunov, nom. nov., based on Dipsacaceae Juss., Gen. Pl.: 194. 4 August 1789, nom. cons.Type: Dipsacus L. (1753).

Escalloniineae Shipunov, nom. nov., based on Escallonieae R.Br. ex DC., Prodr. 4: 2. Late September 1830.-Type: Escallonia Mutis ex L.f. (1782).

Eupteleineae Shipunov, nom. nov., based on Eupteleanae Doweld, Tent. Syst. Pl. Vasc.: xxv. 23 December 2001.-Type: Euptelea Siebold \& Zucc. (1840).

Gunnerineae Shipunov, nom. nov., based on Gunneraceae Meisn., Pl. Vasc. Gen.: Tab. Diagn. 345, 346, Comm. 257. 13-15 February 1842, nom. cons.-Type: Gunnera L. (1767).

Lardizabalineae Shipunov, nom. nov., based on Lardizabaleae DC., Prodr. 1: 95. January (med.) 1824.Type: Lardizabala Ruiz \& Pav. (1794). 
Linineae Shipunov, nom. nov., based on Lineae Rchb., Fl. Germ. Excurs. 2(2): 830, 831. 1832.-Type: Linum L. (1753).

Nelumbonineae Shipunov, nom. nov., based on Nelumboneae DC., Syst. Nat. 2: 43. Late May 1821.—Type: Nelumbo Adans. (1763).

Petrosaviineae Shipunov, nom. nov., based on Petrosavianae Doweld, Tent. Syst. Pl. Vasc.: 1x. 23 December 2001.-Type: Petrosavia Becc. (1871).

Picramniineae Shipunov, nom. nov., based on Picramniales Doweld, Tent. Syst. Pl. Vasc.: xxxviii. 23 December 2001.- Type: Picramnia Sw., nom. cons. (1788).

Piperineae Shipunov, nom. nov., based on Piperaceae Giseke, Prael. Ord. Nat. Pl.: 123. April 1792, nom. cons.-Type: Piper L. (1753).

Rhabdodendrineae Shipunov, nom. nov., based on Rhabdodendrales Doweld, Tent. Syst. Pl. Vasc.: xli. 23 December 2001.-Type: Rhabdodendron Gilg \& Pilg. (1905).

Rhamnineae Shipunov, nom. nov., based on Rhamnaceae Juss., Gen. Pl.: 376. 4 August 1789 (Rhamni), nom. cons.-Type: Rhamnus L. (1753).

Rhizophorineae Shipunov, nom. nov., based on Rhizophoraceae Pers., Syn. Pl. 2: 2. November 1806, nom. cons.-Type: Rhizophora L. (1753).

Schisandrineae Shipunov, nom. nov., based on Schisandraceae Blume, Fl. Javae 32-33: 3. 25 June 1830, nom. cons.-Type: Schisandra Michx., nom. cons. (1803).

Winterineae Shipunov, nom. nov., based on Winteranae Doweld, Tent. Syst. Pl. Vasc.: xxiii. 23 December 2001.-Type: Wintera Murray (1784).

\section{References}

Angiosperm Phylogeny Group [Bremer, B., Bremer, K., Chase, M.W., Fay, M.F., Reveal, J.L., Soltis, D.E., Soltis, P.S. \& Stevens, P.F. (comp.)] III (2009) An update of the Angiosperm Phylogeny Group classification for the orders and families of flowering plants: APG III. Botanical Journal of the Linnean Society of London 161: 105-121.

Shipunov, A.B. (2003) The system of flowering plants: synthesis of classical and molecular approaches. Zhurnal Obshchei Biologii 64: 501-519. [In Russian, English abstract].

Takhtajan, A.L. (1997) Diversity and classification of flowering plants. Columbia University Press, New York.

Takhtajan, A.L. (2009) Flowering plants. Springer, New York. 